\title{
CLINICAL, IMAGING AND CYTOLOGICAL DIFFERENCES BETWEEN PALPABLE \\ AND NON-PALPABLE THYROID NODULES
}

J. Nunes e Silva1', S.Paiva², C.Ribeiro², M.Melo², L.Gomes², M. Bastos², F.Carrilho²

Endocrinology, Diabetes and Metabolism Department, Portuguese Armed Forces University Hospital 2. Endocrinology, Diabetes and Metabolism Department, CHUC

\section{Introduction}

The prevalence of palpable nodules varies between 3-7\% and nodules diagnosed by ultrasonography between $20-76 \%$. According to the American Thyroid Association's guidelines it isn't recommended to perform routine thyroid ultrasound for thyroid nodules diagnosis, unless they are palpable or there are any risk factors

\section{Objective}

To evaluate sonographic and cytological differences between palpable and non-palpable thyroid nodules

\section{Methods}

Evaluation of patients referred to perform Fine Needle aspiration Cytology (FNAC) in an Endocrinology department. Clinical evaluation was performed by the same doctor without access to clinical or ultrasound information, characterizing if they had palpable nodules and where they were located. Ultrasound and FNAC were made after clinician evaluation.

\section{Results}

186 Nodules of 139 patients

$85,6 \%$ female

$43 \%$ were palpable nodules

19 nodules didn't perform FNAC, because

didn't met criteria

Figure 1: Patient's and nodules caracterization

\begin{tabular}{|c|c|c|c|c|}
\hline & Total (\%) & $\begin{array}{c}\text { Non- } \\
\text { palpable }\end{array}$ & Palpable & P value \\
\hline $\begin{array}{c}\text { Non } \\
\text { diagnostic }\end{array}$ & $6,5 \%$ & 9 & 2 & 0.122 \\
\hline Benign & $81,4 \%$ & 73 & 59 & 0.043 \\
\hline FLUS & $8,9 \%$ & 8 & 7 & 0.133 \\
\hline $\begin{array}{l}\text { Folicular } \\
\text { neoplasm }\end{array}$ & $1,2 \%$ & 0 & 2 & 0.163 \\
\hline $\begin{array}{l}\text { Suspicious for } \\
\text { malignancy }\end{array}$ & $1,2 \%$ & 1 & 1 & 0.284 \\
\hline Malignancy & $0,6 \%$ & 0 & 1 & 0.367 \\
\hline
\end{tabular}

\begin{tabular}{|c|c|c|c|}
\hline & Non-Palpable & Palpable & P value \\
\hline $\operatorname{Sex} F(n)$ & $68(80)$ & $89(106)$ & 0,325 \\
\hline Age (years) & 57,44 & 57,86 & 0,451 \\
\hline $\begin{array}{l}\text { Location } \\
\text { Superior } \\
\text { Middle } \\
\text { Inferior }\end{array}$ & $\begin{array}{l}18 \\
21 \\
41\end{array}$ & $\begin{array}{l}62 \\
16 \\
28\end{array}$ & $\begin{array}{c}0,034 \\
0,256 \\
0,03\end{array}$ \\
\hline $\begin{array}{l}\text { Localização } \\
\text { - Anterior }\end{array}$ & 37 & 73 & 0,087 \\
\hline Size (mm) & 16,94 & 23,36 & 0,032 \\
\hline $\begin{array}{l}\text { Characteristics } \\
\text { - Solid } \\
\text { - Mixed } \\
\text { - Cyst }\end{array}$ & $\begin{array}{l}16 \\
52 \\
12\end{array}$ & $\begin{array}{l}16 \\
67 \\
23\end{array}$ & $\begin{array}{l}0,214 \\
0,256 \\
0,162\end{array}$ \\
\hline $\begin{array}{l}\text { Hypoechoic } \\
\text { Isoechoic } \\
\text { Hyperechoic }\end{array}$ & $\begin{array}{c}61 \\
8 \\
11\end{array}$ & $\begin{array}{l}84 \\
14 \\
13\end{array}$ & $\begin{array}{l}0,436 \\
0,276 \\
0,384\end{array}$ \\
\hline $\begin{array}{c}\text { infiltrative } \\
\text { margins }\end{array}$ & 16 & 23 & 0,462 \\
\hline microcalcifications & 7 & 12 & 0,385 \\
\hline
\end{tabular}

Table 1: Sonographic characterization of palpable and non-palpable nodules

\section{Discussion/Conclusion}

The palpable nodules were only $43 \%$ of nodules observed by ultrasound. They were larger and were located more superiorly. Non-palpable nodules were more frequent benign cytological results ( statistically significantly ), however malignancy rate between the two types of lesions was similar despite the limitations of the study. Although there is no formal indication for screening non-palpable nodules when they are diagnosed, they have to have an evaluation and follow-ups similar to palpable nodules . 